\title{
El gobierno local en Alemania: continuidad y cambio
}

\section{H ellmut Wollmann*}

El presente artículo consta de dos partesl.

En primer lugar, se realizará un bosquejo de la función del Gobierno Local en el sistema intergubernamental de Alemania.

En segundo lugar, se tratarán las dos corrientes de reformas (N PM o N ew Public M anagement - la reforma administrativa guiada- y la reforma de la democracia local).

\section{La dispersión} intergubernamental en los ámbitos de la toma de decisiones del cambio institucional

En los Estados unitarios o centralistas como el Reino Unido y N ueva Zelanda, aunque también, en menor grado, en los países escandinavos, el poder en la toma de decisiones relacionadas con el cambio institucional descansa predominantemente en el nivel del gobierno central. Por el contrario, ese poder se halla ampliamente disperso en el sistema federal y descentralizado de Alemania, operando tanto vertical como horizontalmente en una multitud de arenas de decisión y ofreciendo la base institucional para un modelo de reforma institucional fragmentado $y$, en la misma lógica, incrementalista.
- D entro de sus competencias constitucionales y legislativas, el nivel federal, en realidad, ejerce una influencia importante en algunos parámetros esenciales del sector público, tales como la privatización del ferrocarril y del sistema postal (anteriormente federales), o la regulación del marco normativo del empleo público y los servicios públicos de las autoridades de los Länder y locales. Sin embargo, como principio constitucional y como norma, el nivel federal no puede interferirse en las cuestiones organizativas, incluyendo la modernización administrativa de los Länder, y dentro de esta misma lógica constitucional, del nivel del gobierno local.

- Los Länder, que históricamente habían reclamado un satus de semisoberanos (Eigenstaatlichkeit) dentro de la estructura federal de dos niveles, son los únicos responsables de sus asuntos organizativos. Este hecho no sólo hace referencia a la Administración de los Länder propiamente dicha, sino también al nivel del gobierno local el cual, por ley constitucional y por principio judicial, es considerado como una parte constitutiva del Länder. La capacidad de actuación institucional de los Länder sobre el nivel local de gobierno incluye la promulgación de las leyes del gobierno local y el diseño de los términos territoriales de los distritos y de los municipios.

- A pesar del poder de los Länder para imponer externamente parámetros institucionales cruciales para su planta institucional, los municipios y los distritos tienen el derecho de decidir sus características organizativas internas de forma autónoma. D e hecho, ese derecho (O rganisations hoheit) se concibe como un elemento esencial del principio de autonomía local (kommunale Selbstverwaltung), 
garantizado por la Constitución Federal. D e este modo, las cuestiones de la reforma administrativa y la modernización son ampliamente decididas por los municipios y los distritos de modo autónomo.

\section{Un nivel federal con un mínimo de funciones administrativas descentralizadas}

Entre tanto, y como resultado de la sigilosa recentralización política que, a través de una gradual acumulación de funciones legislativas y de elaboración de políticas, se ha producido en la República Federal desde su establecimiento en 1949, cuando se diseñó como un sistema federal también políticamente descentralizado, las funciones administrativas, especialmente las de tipo ejecutivo, del sistema federal de gobierno, se mantuvieron de modo llamativamente reducido. A diferencia del sistema federal de los EEUU, en el que el Gobierno Federal puede disponer de oficinas regionales y locales propias, con el fin de implementar las políticas y los programas federales o de guiar y supervisar su implementación por parte de las agencias estatales, en el sistema federal alemán al nivel del Gobierno Federal se le excluye constitucionalmente de la titularidad de cualquier oficina local o regional y, por tanto, difícilmente posee una estructura ejecutiva, excepto en el pequeño conjunto de responsabilidades típicamente federales constitucionalmente enumeradas, tales como las aduanas, el servicio militar y la policía fronteriza. Por lo tanto, la implementación de la legislación y de las políticas federales (aś́ como las crecientes previsiones normativas y programas de financiación de la Unión Europea) recae casi enteramente sobre los Länder.

Este peculiar sistema constitucional que separa, de forma estricta, las funciones legislativas y de elaboración de políticas en el nivel federal de la función ejecutiva en el nivel de los Länder, puede interpretarse en términos generales como una variante radical de la agencialización o mandato, en la que el nivel federal (como el «principal») tiene poco poder formal y de control sobre los L änder (como sus «agentes» de implementación), excepto en la referente al principio de la lealtad federal mutua (Bundestreue) y a los usos del federalismo cooperativo, que son considerados como las reglas básicas del juego intergubernamental federal.
D esde el punto de vista organizativo, el nivel de G obierno Federal está compuesto por los ministerios federales y unas 160 agencias y organizaciones federales. Éstas operan - en lo que puede nuevamente ser entendido como una forma de agencialización- bajo la supervisión general de los ministerios federales, dentro de un amplio espectro de funciones subsidiarias, principalmente no ejecutivas, sin oficinas «subfederales» de su titularidad (Benz/G OETZ, 1996: 6). El ferrocarril federal y los sistemas postales constituyeron una gran presencia federal en el nivel te rritorial más bajo (intensiva en recursos humanos), antes de que fueran privatizados a principios de los noventa y su personal fuera apartado de la plantilla federal.

En 1994, después de que se efectuase la privatización del fe rrocarril y de los correos y telecomunicaciones federales, el personal federal constituía el 11,9 por ciento del número total de empleados públicos, que es significativamente pequeño para los estándares internacionales, obviamente en comparación con los países unitarios (tales como N ueva Zelanda, con un 89,7 por ciento; Francia, con un 48,7 por ciento; y el Reino Unido con un 47,7 por ciento), pero también en comparación con otros países federales (tales como los EE.UU, con un 15,2 por ciento y Canadá, con un 17,1 por ciento). Como la plantilla federal es, por tanto, excepcional mente escasa y como los ministerios, las agencias (en cierto modo «mandatarias») y las unidades federales no poseen casi funciones ejecutivas, la «condición inicial» para una próxima modernización es, por tanto, significativamente diferente de la de los países con una estructura de gobierno central de mayor peso.

\section{El nivel del Länder como pilar del sistema administrativo intergubernamental, en tanto que deja la mayor parte de las funciones ejecutivas en las autoridades locales}

M ientras que el papel político de los Länder en la legislación y en la elaboración de las políticas públicas ha ido disminuyendo como resultado de la silenciosa recentralización pol ítica de la República (y continuará haciéndolo así, como en el caso de la transferencia de las competencias nacionales a la Unión Europea), aquellos poseen, en la relación federal/Länder, intacto todo su 
«monopolístico» control de la implementación de la legislación, políticas y programas de los niveles federal, del Länder, y cada vez más también del nivel europeo. D entro de cada Land, sin embargo, las funciones administrativas están ampliamente desconcentradas - en términos de Ciencia de la Administración - y en parte también (políticamente) descentralizadas en un tradicional sistema de tres niveles: en el nivel central del Land opera su gobierno con sus departamentos y un sistema (en cierto modo, «agencializado») de agencias centrales gestionando funciones políticas auxiliares. En el nivel intermedio, en una tradición administrativa que se remonta al siglo XIX y a la «importación» institucional de los dé partements franceses, los distritos administrativos (Regierungsbezirke) se encargan de lo que ha sido denominado como función de «encauzar» 0 «impulsar» (Trichter-, Bündelungfunktion), coordinar regionalmente la implementación de las políticas sectoriales y los programas, así como en supervisar y asesorar a las autoridades locales (FüRST, 1996). Los distritos administrativos están liderados por un funcionario público (al estilo del prefecto francés) de signado por el gobierno del Land, ostentando el prestigioso título de «presidente gubernamental» (Regierungspräsident).

En el nivel local, sin embargo, sólo se están ejerciendo directamente por el Länder unas pocas actuaciones públicas, a cargo de las unidades administrativas específicas (Sonderbehörden). En cambio, como se explica a continuación, «a parte del león» de las actuaciones públicas y ejecutivas se descarga en las autoridades locales, esto es, en la administración del distrito (krés) y municipal ( $G$ emende, Stadt). Este hecho supone un contraste chocante, por ejemplo, con Francia, donde incluso desde la descentralización practicada a principios de los ochenta, las principales tareas administrativas continúan siendo gestionadas por oficinas de la Administración periférica (services extérieurs), subordinadas directamente a los ministerios centrales de París. Länder:

Como muestra del perfil funcional de la Administración del

- El 45 por ciento de su personal está empleado en educación, ciencia y asuntos culturales (señalando el hecho de que los colegios y universidades son casi de completa titularidad estatal, de acuerdo con la tradición continental europea, y que el ámbito de las materias educativas y culturales son una prerrogativa - Kulturhoheit- del Länder).

- El 12 por ciento en policía (constituyendo la policía otra competencia estatal clásica en la tradición alemanal continental europea y recayendo dicha competencia en el Länder).

- Y (solamente) el 13 por ciento trabaja en la Administración Pública del Länder en sentido estricto.

Dentro de esteúltimo grupo, se incluye personal:

- En el nivel central (con funciones en la elaboración de políticas públicas).
- En el nivel intermedio (con funciones de supervisión y de coordinación).

- En el nivel local (con un comparativamente restringido margen de funciones ejecutivas).

Esta circunstancia muestra las diferentes condiciones de inicio y las necesidades para las reformas administrativas en cada uno de estos niveles intra-Länder.

Finalmente se ha de observar que los empleados públicos del Länder suman el 45 por ciento del empleo de todo el sector públiCo, estando dicho porcentaje significativamente por encima de los EE.UU. (con un 22,6 por ciento), aproximadamenteigual a Cana dá (con un 44,1 por ciento) y muy por debajo de Australia (con un 73,3 por ciento)2. En la interpretación de estas cifras se ha de recordar que en Alemania la educación y la policía han sido tradicionalmente competencias estatales, con su correspondiente personal integrando un amplio segmento de la plantilla de los Länder.

\section{Unas autoridades locales desempeñando la «parte del león» de las tareas administrativas}

Bajo la fórmula de la competencia general, constitucionalmente garantizada, que se remonta al reconocimiento de la autonomía local a principios del siglo XIX, «os municipios y las distritos tienen el derecho y la obligación de actuar, bajos su propia responsabilidad, dentro del marco de la ley, en todas las materias re levantes para la comunidad local» (de acuerdo con el artículo 28 de la Constitución Federal de 1949); este hecho supone constitucional e institucionalmente un modelo de autonomía local (kommunale Selbstverwaltung) multifuncional.

Además, en una tradición institucional (de doble función), que también data del siglo xIx (y que constituye una peculiaridad de la tradición germano-austriaca de administración y de gobierno local y que rige esencialmente el modelo multifuncional), a los distritos y a los municipios se les encarga el desempeño de tareas administrativas delegadas por el Estado (W o LLM ANN, 2000b: 116 ff.). M ientras que la dirección de las competencias locales propias y de las competencias delegadas por el Estado se hall an horizontalmente integradas y fundidas dentro de las funciones de las autoridades locales, sus modalidades operativas han sido clásicamente diferentes con respecto a las relaciones verticales entre Estado (Land) y gobierno local. M ientras que en el desarrollo de las com- 
petencias local es propias las autoridades locales actúan bajo supervisión del Estado tan sólo en los aspectos de legalidad (dentro del marco de la ley) de sus actividades (Rechtskontrolle), en el caso de las competencias delegadas, el control por el Estado va más allá y también afecta a los contenidos sustantivos (Fachaufsicht)3.

Entre las materias tradicionalmente propias de la autonomía local se incluyen ampliamente las siguientes:

- Planificación local (clasificación de los suelos, planificación del desarrollo urbano).

- Construcción y mantenimiento de las infraestructuras urbanas (vías públicas, aguas residuales, etc.) y equipamientos públicos (de hecho, dos terceras partes de todas las inversiones en capital público se efectúa por las autoridades locales).

- Funciones de política social (partiendo de su histórica responsabilidad en la aplicación de la ley de pobres, los gobiernos locales han sido administrativamente, y también desde el punto de vista financiero, responsables del sistema de asistencia social y de la provisión, 0 al menos de hacer posibles los servicios sociales del gobierno local a través de un abanico de «servicios de Administración pública prestacional» (Leistungsverwaltung).

- Un creciente número de políticas y tareas (rehabilitación urbana, promoción económica, desempleo, protección medioambiental) asumidas por el gobierno local.

Entre las tareas delegadas se ha al udido ampliamente el ámbito de las competencias administrativas referentes al «mantenimiento del orden público» (Ordnungsverwaltung), esto es, la aplicación y fortalecimiento de las previsiones legales en materia de inspección de edificios, protección medioambiental así como en permisos de vehículos, permisos de conducir, Registro Civil y similares. Se ha estimado que entre el 70 y el 85 por ciento de la legislación federal y de los Länder (así como el creciente cuerpo legislativo comunitario) está siendo implementada por las autoridades locales.

D esde el punto de vista del excepcionalmente amplio conjunto de competencias del gobierno local se necesita subrayar algunas salvedades:

- A pesar del amplio espectro de responsabilidades locales en política social, la prestación de los servicios sociales de carácter personal (jardines de infancia, centros de ancianos, etc.) se ha dejado, de acuerdo con el tradicional principio de «subsidiariedad», en manos de un amplio número de organizaciones no públicas, no lucrativas (tipo $0 \mathrm{NG}$ ) de bienestar social (freieWohlfahrtsverbände) (BöN KER/ W OLLMANN , 2000: 337), en tanto queel gobierno local se dedica a ejercer (en términos de N ueva G estión Pública) la función de «supervisión» y comprometiéndose a prestar servi- cios a través de su propio personal únicamente en aquellos casos en que las $0 \mathrm{~N}$ Gs no los asuman. De nuevo este he cho difiere significativamente de la práctica del Reino Unido y de los países escandinavos, con el predominio del personal de la Administración local en la prestación de los servicios sociales de carácter personal.

- Además, debe recordarse que en la tradición alemana (al igual que en la continental europea) la educación y la policía se consideran competencias estatales; consecuentemente la implicación del gobierno local en los colegios de primaria y secundaria consiste tan sólo en la dimensión técnica de la construcción de colegiosy de su mantenimiento (incluyendo al personal técnico, tal como jardineros, limpiadores, etc.), en tanto queel empleo de los profesores, currículo y demás corresponde claramente a las autoridades de los Länder.

La esfera funcional de las actividades del gobierno local se ve reflejada en la composición de su personal4:

- Por término medio, un 14 por ciento se encuentra empleado en Administración social.

- Un 7 por ciento en prestación de salud, deportes y equipamientos de ocio.

- Un 10 por ciento en el mantenimiento de colegios y escuelas nocturnas de adultos.

- Un 7 por ciento en las funciones de «mantenimiento del orden público» (0 rdnungsverwaltung).

- Un 8 por ciento en actividades relacionadas con las tareas de promoción económica.

- Un 15 por ciento en Administración local general.

El listado expuesto de grupos de tareas y de plantillas de personal adscritas a aquéllas hace referencia a la heterogeneidad (planificación, inversiones de capital, aplicación de la ley, política sectorial, etc.) de las funciones del gobierno local y en los necesariamente variados puntos de partida y estímulos para la reforma administrativa.

\section{El generalismo multifuncional de base territorial frente ala especialización funcional}

La institucionalización del sistema administrativo intergubernamental de Alemania se ha guiado tradicionalmente por el principio de la territorialidad descentralizada multifuncional, 
esto es, asignando el máximo de tareas a los distritos (Landkreis) y a los municipios (Gemeinden, Städte) y poseyendo una Administración territorialmente «unitaria» (Einheit der Verwaltung) en lo que se ha denominado «a orientación histórica (de la Administración alemana) hacia el territorio» (Frido WAGEN ER citando a FÜRST, 1996: 120). Este hecho descansa, obviamente, en la asunción de que, en la controversia entre una organización de un solo fin frente a la organización de múltiples fines (PoLLITT) BOU CKAERT, 2000: 77 y ss.), la Administración de vocación ge neralista, desde una base territorial «unitaria», está más capacitada que las unidades funcionalmente fragmentadas de una sola función para hacer frente al problema de la coordinación representado por la implementación de diferentes, y a menudo conflictivas, políticas y tareas sectoriales (PETERS, 1998).

La proyección del principio de territorialidad fue confirmada y reforzado por la ola de reformas territoriales que los Länder lle varon a cabo a mediados de los sesenta y setenta, fusionando tanto distritos como municipios; con variaciones en la escala de las fusiones entre los Länder, el número de distritos se redujo en el ámbito nacional de una totalidad de 428 a 237 y en el caso de los municipios, de 24.000 a 8.400 (W OLLM AN N, 2000b: 120). La racionalidad que se hallaba detrás de las reformas territoriales iba ampliamente dirigida a incrementar la base territorial del gobierno local para mejorar las funciones de planificación y coordinación y conseguir economías de escala. Como un nuevo paso lógico, se emprendieron reformas funcionales (Funktionalreformen) para transferir nuevas competencias públicas, tradicionalmente desempeñadas por la Administración del Estado, a las autoridades locales. Por lo tanto, el ámbito de las oficinas de una sola función de la Administración del Estado (Sonderbehörden) se vio reducido todavía más.

\section{La proyección del perfil político del gobierno local en el esquema intergubernamental}

El status del gobierno local en el esquema intergubernamental se ha caracterizado por una llamativa contradicción. Por una parte, un significativo cuerpo de previsiones constitucionales y legales tiende a adscribir al gobierno local un statusinferior (políticamente) dentro del sistema federal, que se construye como un sistema de dos estratos, compuesto por los niveles federal y de Länder. La interpretación jurídica conservadora fue tan lejos como para considerar al gobierno local como una parte constitutiva de la administración del Länder y a la autonomía local como una «Administración indirecta del Estado» (mittelbare Staatsverwaltung). Por otra parte, un creciente número de previsiones constitucionales y legales, así como profundos cambios políticos y funcionales en la relación (intergubernamental) externa y en la articulación institucional interna del gobierno local, han fortalecido significativamente la posición del gobierno local como un sistema político por derecho propio dentro de la estructura intergubernamental de la República Federal. Algunos de estos factores podrían destacarse brevemente:

- Según la Constitución Federal (artículo 28), la democracia local, en cuanto consta de consejos electos, ha sido re conocida de forma explícita y garantizada en pie de igualdad con los Parlamentos de los Länder, otorgándole, de este modo, una presencia constitucional por derecho propio. D espués de 1945, como reacción a la eliminación de la democracia por el régimen nazi, se le otorgó al ámbito local una base constitucional y normativa superior a la de otros países europeos con un pasado democrático sin rupturas y menos tortuoso.

- En la percepción de la población local los municipios (y en menor grado de los distritos) han sido aceptados, tanto política como emocionalmente, como las arenas de la de mocracia local y las sedes de la identidad local. Pueden considerarse como indicadores de tal «localismo», de acuerdo con los estándares internacionales, una alta participación en las elecciones locales (en torno a un 70 por ciento de media) y la resistencia que se ofreció (en algunos casos con éxito) por parte de los habitantes locales frente al proceso de fusión municipal durante los sesenta y setenta. M ientras puede observarse un «localismo» similar, tanto desde el punto de vista político como emocional, en Francia, éste parece hallarse, a causa de diversas razones, ampliamente ausente en el Reino Unido (véase Wo LLM ANN, 2000b: 110).

- El status político del gobierno local se ha visto fortalecido también por su «politización» interna en varios aspectos. En primer lugar, desde los años cincuenta los partidos políticos nacional es han «ocupado» crecientemente la arena local para la competición política. En segundo lugar, en términos políticos y funcionales, los ajustes de poder en el nivel local se han redimensionado por la uparlamentarización» de los consejos electos locales y, de forma diferente de acuerdo con la específica ley de gobierno local aprobada por el Länder, por el fortalecimiento del alcalde, (particularmente si es elegido directamente) en su papel de político y de líder administrativo («jefe ejecutivo») de los municipios (y de manera similar en los distritos). 
En resumen, habría que destacar que el sistema intergubernamental administrativo de Alemania se caracteriza, por lo tanto, por un alto grado de territorialización descentralizada de las funciones administrativas para ser desempeñadas por un gobierno local tanto funcional (multi-función, «función dual», «unitaria») como políticamente fuerte. A causa de la combinación de poderosos elementos funcionales y políticos, el gobierno local de Alemania puede, siguiendo a H ESSE/SH ARPE, 1990, catalogarse dentro del modelo de gobierno local «del Centro y N orte de Europa» (en contraste con el modelo francés con una dimensión política fuerte, pero con una dimensión funcional débil, o con el modelo anglosajón con una dimensión funcional fuerte, pero con una dimensión política débil (véase también W o LLM AN N , 2000b: 223 $\mathrm{ff}$.). D esde el punto de vista del peso funcional (no desde el contenido mínimo del tradicional y el latente incremento de responsabilidades de la «doble función») podría llegar a hablarse de un tipo de gobierno local «germano-austriaco» (W OLLM AN N, 2000b: 125).

\section{Las dos tendencias de reforma que afectan al nivel del gobierno local}

\section{La reforma administrativa}

Nivel de gobierno local

D esde principios de los noventa - por delante del Länder y ciertamente muy por delante del nivel federal- el nivel de gobierno local ha experimentado una tendencia de modernización del sector público bajo el lema del «modelo de nueva dirección» (N eues Steuerungsmodell, N SM ), que fue concebido y difundido por la KGSt, una agencia de consultoría de fundación municipal sin ánimo de lucro (para más detalles ver W OLLM AN, 2000c: 925 ff.). De hecho, el rápido crecimiento de la N ueva Gestión Pública en la retórica y conceptualización predominantes dentro del discurso de la modernización local y en su aplicación, puede atribuirse claramente a la KGSt y a la incesante secuencia de sus informes y experiencias en los que, recurriendo especialmente a los términos empleados entonces en la ciudad holandesa de Tilburg, el mensaje de la Nueva Gestión Pública se explicaba con detalle.

M ientras tanto, la N ueva Gestión Pública había producido un impacto significativo en la modernización administrativa en un creciente número de municipios y distritos, primero en Ale mania 0 ccidental y, con una demora en el tiempo por causa del proceso de unificación y transformación, también en la Alemania del Este. La tendencia de cambio institucional alcanzó más velocidad, al hilo de la emergencia del debate y de la disposición para la modernización, en tanto que los conceptos «tradicionales» de la reforma administrativa que se remontaban a las fases más tempranas de ésta, fueron resucitados y se les dio un nuevo impulso. Esta gran mezcolanza de conceptos de la N ueva G estión Pública (y el paraguas estratégico ofrecido por ellos) con los conceptos de la reforma administrativa «tradicional» es la que imbuye el actual proceso de modernización en muchos municipios y distritos, con sus dinámicas destacables (y aparentemente duraderas).

- En la mayor parte de los municipios y distritos que se han embarcado en la modernización de la Nueva G estión Pública se ha puesto gran énfasis, bajo la presión del ajuste presupuestario, en los instrumentos microeconómicos de aquélla, que prometen una mejor relación coste-eficiencia y una reducción de costes, cuando no una reducción del personal, a modo de presupuesto global en combinación con responsabilidad de gestión descentralizada (dezentrale Ressourcenverantwortung). D ebe observarse que bastante a menudo el nuevo presupuesto ha degenerado en una especie de «fijación de techos de coste» y movimientos de «contención de gasto».

- M ientras la fijación, peculiar en la primera versión del autoritario conjunto de herramientas de Nueva G estión Pública de la KG St, de la definición de «productos» como información clave para el sistema entero, se ha manifestado como un (costoso) fracaso, se han introducido términos menos ambiciosos y más realistas de contabilidad de costes (Kosten-Leistungs-Rechnung) en un creciente número de municipios y distritos, y este hecho ha producido como resultado una «cultura de coste-eficiencia» que ha echado raíces en un terreno administrativo donde todavía prevalecía la tradicional «cultura de regulación legal».

- Junto a los componentes de la N ueva Gestión Pública (el empleo de éstos a menudo permanece en su fase inicial y paralizado por las presiones de reducción de coste) se han emprendido muchas buenas medidas de reforma administrativa «tradicional» en el contexto del emergente debate de la modernización. Puesto que los conceptos de reforma «tradicional» se aplican a menudo a políticas y competencias sectoriales específicas (tal es como la aplicación de la legislación sobre la edificación en los procedimientos de licencia de obras, en la administración social, etc.), son desarrollados y transmitidos por concomitantes constelaciones de actores profesionales dentro de la Administración. 


\section{Introducción de procesos de democracia directa en la política local}

En relación con el nivel de gobierno local debiera mencionarse otra poderosa corriente que ha impactado y ha transformado su estructura político-administrativa, tradicionalmente marcada por el predominio de los principios de la democracia representativa cuando, a principios de los noventa, los Länder procedieron en una llamativa secuencia de movimientos legislativos a introducir procesos de democracia directa, es decir, referéndum locales (obligatorios) y elección directa de los jefes ejecutivos locales (véase W ollm An, 2000 a 2000c: 928, con referencias).

- Si bien hace poco Baden-W ürttemberg era el único Land en ofrecer, desde mediados de los cincuenta, tales proce dimientos, se han introducido referéndum locales (obligatorios) desde principios de los años noventa en casi todos los Länder. Haciéndose eco y respondiendo al movimiento cívico democrático que jugó un papel importante en el derrumbamiento del régimen comunista, la Ley municipal aprobada en mayo de 1990 por el Parlamento democráticamente el egido de la todavía existente República D emocrática Alemana estipulaba referéndum locales obligatorios. M ediante una amplia serie de leyes casi todos los Länder siguieron el ejemplo. Aun cuando la población local puede abordar, vía referéndum, todas las «cuestiones locales», las cuestiones del presupuesto local y la organización interna de la Administración local, como importantes excepciones, no pueden ser objeto de un re feréndum local.

- En otro amplio cambio institucional, los Länder, a principios de los noventa, en unos movimientos legislativos llamativamente congruentes, introdujeron la elección directa del (monocrático) alcalde (y también del presidente de los distritos: el Landräte); además, algunos Länder también han establecido procedimientos para cesar a los alcaldes y al Landräte por referéndum local. En las leves municipales aprobadas después de 1945, sólo dos Länder (Baden-Württemberg y Baviera) habían presentado modelos de alcaldes por elección directa ( «fuertes», siguiendo el correspondiente modelo de los EE.UU.), mientras otros Länder adoptaron una gran variedad de modelos. Concretamente en los Länder de Alemania del Este, la adopción de la elección directa de los alcaldes estaba primordialmente motivada por razones democráticas, mientras que en algunos Länder de Alemania 0 ccidental residía mayormente en la intención de fortalecer la capacidad «gerencialista» y la autoridad de los jefes de los ejecutivos locales. En algunos Länder (especialmente de la Alemania del Este) la modalidad de elección directa de los alcaldes se complementaba con la introducción de procedimientos de destitución por los cuales a la población local se le otorgaba el derecho de destituir al alcalde por referéndum local (es significativo mencionar que en el Land de Brandenburgo en la Alemania del Este, desde fines de 1993, cuando se aprobó la nueva legislación, habían perdido sus puestos en torno a jun diez por ciento! de los alcaldes a tiempo completo como resultado de procesos de referéndum de destitución).

Con la introducción de estos procedimientos de democracia directa, el gobierno local de Alemania se ha colocado a la cabeza de la carrera de los países europeos, junto con Suiza, como clásica patria de la democracia directa, y más recientemente Italia. EI impacto de estas novedades institucionales, particularmente la de la elección directa de los jefes de los ejecutivos locales, está llamado a ser significativo, en la medida en que el perfil político del gobierno local como contexto y el marco de referencia de las reformas administrativas se han visto acentuadas y la responsabilidad política de los jefes ejecutivos (directamente elegidos) se ha fortalecido.

\footnotetext{
* Profesor de la Universidad Humboldt, de Berlín.

La traducción española de la versión orginal inglesa ha sido realizada por Pedro Luis Pérez Guerrero, Profesor del Área de Ciencia Política y de la Administración de la Universidad de Alicante.

$1 \mathrm{El}$ artículo forma parte de W OLLM AN N (2001).

2 D atos tomados de PollitT/BOuckaerT, 2000: 44 (al igual que los de 1994).

3 El funcionamiento del modelo dual de relaciones Estado/gobierno local exhibe al guna ambivalencia visible. Por una parte, se puede argumentar que en la delegación de funciones administrativas del Estado en combinación con la supervisión sustantiva que va con ella (Fachaufsicht), el Estado adopta un manejable control jerárquico sobre los gobiernos locales, el cual recae sobre la dirección de las fun-
}

ciones del gobierno local y, por tanto, tiende a «estatalizarlas» (verstaatlichen). Por otra parte, también es, de otro modo, cuestionable que las competencias delegadas del Estado sean propensas a ser «municipalizadas» (kommunalisieren), en la medida en que son ejecutadas por la Administración local bajo la dirección del jefe ejecutivo local (esto es, desde hace poco en todos los Länder, bajo la dirección de un alcalde elegido directamente o del jefe de la Administración del distrito) y están, por tanto, expuestas a un contexto político incluso más intenso. Existe evidencia empírica de que el efecto «municipalizador» se ha hecho sentir crecientemente.

4 Cifras de 1993 para los municipios y distritos de Alemania 0 ccidental, cifras tomadas de Lo Ren Z/W OLLMANn, 1999: 509. 


\section{Bibliografía}

Benz, Arthur y Goetz, Klaus H. (1996). «T he German Public Sector: National Priorities and the International Reform Agenda", en BENZ, Arthur y GOETZ, Klaus H. (eds.), A N ew German Public Sector? Reform, Adaptation and Stability, Aldershot, $H$ ants: D artmouth Publishers, pp. 1-26.

Bönker, Frank y Wollm ann, H ellmut (2000). «T he Rise and Fall of a Social Service Regime: M arketisation of German Social Services in H istorical Perspective», en Hellmut W o lLm an n/Eckhard H (eds.), Comparing Public Sector Reform in Britain and Germany. Key traditions and trends of modernisation, Aldershot, etc.: Ashgate, pp. 327-350. Western Europe, 0 xford.

FüRST, D ietrich (1996). «The Regional Districts in Search of a New Role», en BENZ, Arthur y G OETZ, Klaus H. (eds.), A N ew German Public Sector? Reform, Adaptation and Stability, Aldershot, H ants: D artmouth Publishers, pp. 119-136.

Lorenz, Sabine y Wollm Ann, H ellmut (1999). «Kommunales Dienstrecht und Personal», en H ellmut W o LLm An n/Roland ROTH (H rsg.), Kommunal politik, O pla den: Leske +Budrich, S. 490-511.

Peters, B. G uy (1998). «M anaging H orizontal Government: The Politics of Coordination», en Goverfance, vol. 76, Summer 1998, pp. 195-311.

Pollitt, Christopher y Bouckaert, Geert (2000). Public M anagement Reform. A Comparative Analysis, 0 xford: University Press.
Wo LLM AN N, H ellmut (1999). «Kommunalvertretungen: Verwaltungsorgane oder Parlamente?», en H ellmut Wollm ann/Roland Roth (H rsg.), Kommunal politik. Politisches Handeln in den Gemeinden, O pladen: Leske + Budrich, S. 50-66.

Wollm An N, H ellmut (2000a). «Comparing Institutional Development in Britain and Germany: (Persistent) Divergence or (Progressing) Convergence?», en H ellmut W ollm an n/Eckhard Sch RöTer (eds.), Comparing Public Sector Reform in Britain and $G$ ermany. Key traditions and trends of modernisation, Aldershot, etc. Ashgate, pp. $1-26$.

W OLLman N, Hellmut (2000b). «T he D evelopment and Present State of Local Government in England and Germany - a Comparison», en Hellmut W OLLM AN N / Eckhard SCHRÖTER (eds.), Comparing Public Sector Reform in Britain and Germany. Key traditions and trends of modernisation, Aldershot, etc., Ashgate, pp. 107-131.

W ollmann, Hellmut (2000c). «Local Government M odernisation in Germany: Stages, discourses, patterns, international perspective», en Public Administration, vol. 78, no 4, pp. 915-936.

W OLLM AN N, H ellmut (2001). «G ermany's trajectory of public sector modernisation: continuities and discontinuities», en Policy $\&$ Politics, vol. 29, no. 2, pp. 151-170. 